\title{
An update on acquired aplastic anemia
}

\section{Opinion}

Acquired Aplastic anemia is a rare bone marrow failure syndrome, characterised in most of the cases by autoimmune destruction of hematopoietic stem cells in the bone marrow. ${ }^{1}$ The activated cytotoxic $\mathrm{T}$ cells in aplastic anemia display various abnormalities, leading to INF-g overproduction. This aberrant IFN-g levels lead to Fas-mediated stem cell death, resulting in replacement of the cellular component of the bone marrow by fat. The result of this replacement is pancytopenia in the peripheral blood. ${ }^{2}$ Several causes have been implicated in the onset of aplastic anemia (secondary aplastic anemia) as pregnancy, drugs (chloramphenicol, non-steroid anti-inflammatory drugs, chemotherapeutic agents), radiation, hepatitis, etc. But in most of cases there is no causative factor identified and aplastic anemia is characterised as idiopathic aplastic anemia. Idiopathic aplastic anemia usually occurs in young people (average age of onset is the second to third decade of life and a small increase in disease incidence occurs also around the fifth to sixth decade). In order to perform the diagnosis of plastic anemia the bone marrow cellularity should be below $30 \%$. Depending on the counts in the peripheral blood idiopathic aplastic anemia can be categorised as very severe, severe, or moderate. In very severe aplastic anemia the absolute neutrophil count is less than 200/ $\mu \mathrm{L}$, the number of platelets below $20,000 \mu \mathrm{L}$ and the absolute number of reticulocytes is below 20,000. In severe aplastic anemia absolute neutrophil count is less than $500 / \mu \mathrm{L}$, while in moderate aplastic anemia absolute number of neutrophils is more than $500 / \mu \mathrm{L}$, but there is a need for transfusion of red blood cells or platelets. ${ }^{1-3}$

\section{Pathogenesis of acquired aplastic anemia}

T cells: In idiopathic aplastic anemia there are increased circulating cytotoxic $\mathrm{T}$ lymphocytes that overproduce interferongamma, which is responsible for the destruction of bone marrow stem cells. These $\mathrm{T}$ lymphocytes over-express the transcription factor T-bet, the hallmark transcription factor for IFN-g production. ${ }^{4}$ The antigens which stimulate these $\mathrm{T}$ lymphocytes and are constantly in an activated state are not yet known. Furthermore there are reduced regulatory $\mathrm{T}$ lymphocytes, which cannot suppress the over-activation of the T cytotoxic lymphocytes. The Th17 cells, the subpopulation of $\mathrm{T}$ cells that normally continue the inflammatory process that Th1 cells start, are also increased in aplastic anemia. In fact, there is an inverse relationship between Th17 and T regulatory cells; the reduced number of regulatory cells is related to the increased Th17 circulating cells. ${ }^{5,6}$ The excessive growth of the interferon-gamma results in destruction of the stem cells in the bone through Fas-induced apoptosis resulting in the replacement of fat and appearance of pancytopenia in peripheral blood.

Telomeres: Approximately one third of the patients with aplastic anemia have shorter telomeres, while only $3-5 \%$ of these patients have mutations in the TERT and TERC genes. ${ }^{7}$ Telomeres are short hexameric repetitive DNA sequences at the end of the chromosomes that shield and protect the chromosomes so that in each cell division genetic material is not lost. Studies have shown that the shorter the telomeres in aplastic anemia patients, the greater the probability these patients have not to respond to immunosuppressive therapy or relapse after treatment. $^{8}$
Volume 4 Issue 2 - 2017

\author{
Elena E Solomou \\ Department of Internal Medicine-Hematology, University of \\ Patras Medical School, Greece \\ Correspondence: Elena E Solomou, MD, PhD, Assistant \\ Professor Internal Medicine-Hematology, University of Patras \\ Medical School, Rion 26500, Greece, \\ Email elenasolomou@hotmail.com, esolomou@med.upatras.gr
}

Received: April 03, 2017| Published: April 06, 2017

Mutations: With new molecular techniques about $20 \%$ of patients with aplastic anemia may have karyotypic abnormalities or mutations. The most frequent karyotypic abnormalities involve chromosome 7 (monosomy 7), trisomy 8, deletion of 5q, 20q deletion and trisomy 1q. Also mutations in the TP53 gene, ASXL1 and DNMT3 are associated with poor prognosis while mutations in PIGA, BCOR and BCORL1 genes are associated with better prognosis. ${ }^{9}$

\section{Clinical presentation}

Patients with aplastic anemia usually present with all the symptoms of pancytopenia. Because of anemia, weakness, fatigue and irritability are observed and symptoms can be so severe that in most of the cases frequent transfusions are required. Because of thrombocytopenia petechiae, bruising and other bleeding events are also present. Severe neutropenia results in bacterial and fungal infections, which could be the first presentation of the disease. No lymphadenopathy or hepatosplenomegaly are present. In a patient presenting with pancytopenia a detailed medical history is essential to rule out other causes of pancytopenia (eg. leishmaniasis, malaria, vitamin B12 or folate deficiency, myelodysplastic syndromes, hypersplenism, other systemic infections, systemic lupus erythematosus, chemotherapeutics or other drugs). In patients aged less than 40years, Fanconi anemia should also be included in the differential diagnosis.

\section{Laboratory findings}

During the diagnosis of aplastic anemia, the anemia is usually orthochromatic macrocytic and also neutropenia and thrombocytopenia are present, as mentioned before. Bone marrow cellularity is very low $(<30 \%)$. Bone marrow biopsy should be obtained from both posterior iliac crests in order to be confident for the correct cellularity and the diagnosis. The classic karyotype technique may not reveal abnormalities, but with newer techniques (i.e. SNP-array karyotype analysis) abnormalities may be present as described above. The presence of a paroxysmal nocturnal hemoglobinuria ( $\mathrm{PNH}$ ) clone should also be tested in these patients, using a peripheral blood sample. The aplastic anemia patients who carry also a PNH clone do not show hemolysis, but usually respond better to immunosuppressive therapy. ${ }^{1,2}$

\section{Differential diagnosis}

All secondary causes of pancytopenia should be excluded. The most difficult is to distinguish aplastic anemia from 
hypoplastic myelodysplastic syndromes and PNH. The hypoplastic myelodysplastic syndromes usually involve older ages, are often present with abnormal karyotype and usually have increased marrow cellularity and peripheral blood counts, compared to aplastic anemia patients. Paroxysmal nocturnal hemoglobinuria is usually diagnosed with Coombs-negative hemolytic anemia, thrombosis at unusual sites and bone marrow failure. ${ }^{10}$

\section{Clonal evolution}

Approximately $20 \%$ of patients with aplastic anemia will evolve during the course of the disease into myelodysplastic syndrome or acute myelogenous leukemia. Despite progress in the pathogenesis and treatment options, this evolution rate remains the same today. ${ }^{3}$

\section{Treatment}

First line treatment option ${ }^{11}$ is allogeneic stem cell transplantation in young patients (less than 40years of age) who have a matched sibling donor. In older patients with an available sibling donor, usually transplantation is not performed because of the increased rate of GVHD and graft failure. Conditioning regimens with fludarabine and cyclophosphamide along with anti-thymocyte globulin are usually used. It should be noted that the stem cell source for transplantation in aplastic anemia should be the bone marrow. In patients older than 40years or in those where no identical sibling donor is available, immune suppression with cyclosporin with anti-thymocyte globulin remains the gold standard. ${ }^{12}$ Cyclosporin is usually continued for at least a year in low doses. Only $60 \%$ of aplastic anemia patients will respond to this treatment option and one third of the patients that initially respond will relapse. After six months of the initial course of anti-thymocyte globulin, a second course can be given to those patients who did not respond. In this second round of antithymocyte globulin, only $30 \%$ of the patients will respond. Recently, Eltrombopag is approved for those patients who do not respond to immunosuppressive treatment with cyclosporine and anti-thymocyte globulin. ${ }^{13}$ Eltrombopag is an oral small non-peptide molecule that binds to the thrombopoietin receptor, but at a different binding site from the endogenous thrombopoietin, hence does not compete with endogenous thrombopoietin for binding on its receptor. It was first approved for chronic idiopathic thrombocytopenic purpura, but when used in refractory to immune suppression aplastic anemia patients, $40 \%$ of these patients showed tri-lineage response. ${ }^{14}$ Additionally, when the drug was discontinued, these patients sustained their response. No increase in clonal evolution was observed with Eltrombopag compared to immune suppression. ${ }^{15}$ Last year the results of a trial using Eltrombopag upfront in treatment-naive aplastic anemia patients were published; when Eltrombopag is used upfront with antithymocyte globulin and cyclosporine a response rate of $95 \%$ was observed. ${ }^{16}$ Clinical trials are ongoing in Europe and in the United States using Eltrombopag upfront with immune suppression and the results so far show that Eltrombopag will be the new player in the first line treatment in aplastic anemia patients. Androgens may show some response in aplastic anemia patients and recently it was shown that can elongate telomeres. The use of androgens with immune suppression remains an option, especially in patients with short telomeres. The use of granulocyte colony stimulating factor (G-CSF) when was first used did not show promising results. In later studies it was shown that it does not increase the rate of evolution to myelodysplasia or leukemia and remains an option.

\section{Summary}

Acquired aplastic anemia is a rare autoimmune disease that needs urgent diagnosis and treatment. The current published data on pathogenesis lead to better understanding on the disease and novel treatment options with the use of Eltrombopag upfront will probably change the therapeutic plan in this complicated disease.

\section{Acknowledgements}

None.

\section{Conflict of interest}

The author declares no conflict of interest.

\section{References}

1. Young NS, Calado RT, Scheinberg P. Current concepts in the pathophysiology and treatment of aplastic anemia. Blood. 2006;108(8):2509-2519.

2. Young NS, Bacigalupo A, Marsh JC. Aplastic anemia: pathophysiology and treatment. Biol Blood Marrow Transplant. 2010;16(1Suppl):S119 S125.

3. Young NS, Scheinberg P, Calado RT. Aplastic anemia. Curr Opin Hematol. 2008;15(3):162-168.

4. Solomou EE, Keyvanfar K, Young NS. T-bet, a Th1 transcription factor, is up-regulated in $\mathrm{T}$ cells from patients with aplastic anemia. Blood. 2006;107(10):3983-3991.

5. Solomou EE, Rezvani K, Mielke S, et al. Deficient CD4+ CD25+ FOXP3 $+\mathrm{T}$ regulatory cells in acquired aplastic anemia. Blood. 2007;110(5):1603-1606.

6. De Latour RP, Visconte V, Takaku T, et al. Th17 immune responses contribute to the pathophysiology of aplastic anemia. Blood. 2010;116(20):4175-4184.

7. Yamaguchi H, Calado RT, Ly H, et al. Mutations in TERT, the gene for telomerase reverse transcriptase, in aplastic anemia. $N$ Engl J Med. 2005;352(14):1413-1424.

8. Scheinberg P, Cooper JN, Sloand EM, et al. Association of telomere length of peripheral blood leukocytes with hematopoietic relapse, malignant transformation and survival in severe aplastic anemia. JAMA. 2010;304(12):1358-1364.

9. Babushok DV, Olson TS, Bessler M. Somatic mutations and clonal hematopoiesis in aplastic anemia. N Engl J Med. 2015;373(17):1673.

10. Killick SB, Bown N, Cavenagh J, et al. Guidelines for the diagnosis and management of adult aplastic anaemia. Br J Haematol. 2016;172(2):187207.

11. Bacigalupo A. How I treat acquired aplastic anemia. Blood. 2017;129(11):1428-1436.

12. Scheinberg P, Young NS. How I treat acquired aplastic anemia. Blood. 2012;120(6):1185-1196.

13. Olnes MJ, Scheinberg P, Calvo KR, et al. Eltrombopag and improved hematopoiesis in refractory aplastic anemia. $N$ Engl J Med. 2012;367(1):11-19.

14. Marsh JC, Mufti GJ. Eltrombopag: a stem cell cookie? Blood. 2014;123(12):1774-1775.

15. Desmond R, Townsley DM, Dunbar C, et al. Eltrombopag in aplastic anemia. Semin Hematol. 2015;52(1):31-37.

16. Townsley DM, Dumitriu B, Scheinberg P, et al. Eltrombopag added to standard immunosuppression for aplastic anemia accelerates count recovery and increases response rates. Blood. 2015;126(23 LBA-2). 